\title{
SIMULATION AND MEASUREMENT STUDIES OF THE VDES SYSTEM'S TERRESTRIAL COMPONENT
}

\author{
Krzysztof Bronk \\ Patryk Koncicki \\ Adam Lipka \\ Dominik Rutkowski \\ Błażej Wereszko \\ National Institute of Telecommunications, Wireless Systems and Networks Department, Gdansk, Poland
}

\begin{abstract}
In the paper, the measurement and simulation results of the VDES (VHF Data Exchange System) terrestrial component are discussed. It is anticipated that VDES will be one of the major solutions for maritime communications in the VHF band and its performance will be sufficient to fulfill the requirements of the e-navigation applications. The process of the VDES standardization (ITU R, IALA) has not been officially completed yet, but substantial amount of technical information about the future system's terrestrial component (VDE-TER) is already available. The paper is divided into three general parts: (a) theoretical presentation of the system's physical layer and the radio channels applicable to $V D E S$, (b) simulation results (BER, BLER, channel delay between two propagation paths and its influence on bit rates) and (c) measurement results (useful ranges, BER). It turned out that in real maritime conditions, the VDES system can offer ranges between 25 and $38 \mathrm{~km}$ for the configurations assumed during the measurement campaign. Those results are generally compliant with the theoretical data in the line-of-sight conditions. In the NLOS scenarios, where fading becomes the dominant phenomenon, the discrepancies between the measurements and the theoretical results were more significant. The obtained results confirmed that VDES provides a large coding gain, which significantly improves the performance of data transmission and increases the bit rate compared to the existing maritime radiocommunication solutions. It should be noted that the results presented in the article were used by the IALA while developing the current version of the VDES specification.
\end{abstract}

Keywords: Maritime radiocommunications, E-navigation, VHF Data Exchange System, VDE-TER, Maritime VHF channels

\section{INTRODUCTION}

NoIn recent years, a significant growth in data transmission systems using maritime radio equipment has become a global trend [9]. Such systems are utilized for many reasons: to improve communications between ships and ports, to increase maritime safety, to protect the natural environment, and also to provide means for the novel concept of so-called e-navigation. One of the projects that deals with the enavigation is EfficienSea2 [4], which is aimed at the development of a hybrid communication system for maritime applications including a new standard of wireless data transmission referred to as VDES (VHF Data Exchange System).
The VDES system is being developed not only to increase the achievable transmission rate but also to improve the availability of data transmission services at sea. To a certain degree, the system is an extension of the existing AIS standard (Automatic Identification System) which was mainly created to ensure an efficient and smooth exchange of navigation data between ships and between ships and coastal entities [10].

In accordance with the ITU-R Recommendation [12], the VDES system should provide an access to the radio link, taking into account the existence of the AIS system and effective utilization of its own spectral resources, and users' needs as well. For this reason, the considered standard will have a lower service priority than the AIS. 
In general, three segments of the VDES system have been defined: terrestrial segment, satellite segment for uplink, and satellite segment for downlink. The following paper is dedicated to the physical layer of the terrestrial part, which is often referred to as VDE-TER.

For the new maritime data exchange standard, the following transmission channels have been assigned:

- Channels 2027 and 2028 for the ASM system (Application Specific Messages),

- Channels 24, 84, 25, 85 with $100 \mathrm{kHz}$ band each for the VDE-TER segment.

In the VDES system, three modulation and coding schemes (MCS) have been defined. The assumed MCSs and three available transmission bandwidths $(25 \mathrm{kHz}, 50 \mathrm{kHz}$ and $100 \mathrm{kHz}$ ) fully define the capabilities of the VDE-TER; their parameters are included in Table 1 [12].

During the work carried out within the framework of the EfficienSea2 project, software implementation of the physical layer for a VDES system's terrestrial component has been developed and the simulation studies based on that software have been executed. As the next step, measurement tests in the maritime environment have been performed to verify the obtained simulation results. It was one of the first ever measurement campaigns of the VDES physical layer (terrestrial component), conducted in its target conditions (i.e. at sea). The findings of both simulation and measurement activities are summarized in the next sections.

The novelty of the paper can be accentuated by the fact that the results discussed below were presented at the IALA forum and had an impact on the current version of the VDES technical specification [12].

Tab. 1. Achievable throughput in the VDE-TER segment

\begin{tabular}{|c|c|c|c|}
\hline \multirow{2}{*}{$\begin{array}{c}\text { Modulation and Coding } \\
\text { Scheme (MCS) }\end{array}$} & \multicolumn{3}{|c|}{$\begin{array}{c}\text { Throughput }(\mathrm{kb} / \mathrm{s}) \\
\text { per bandwidth }\left(\mathrm{RAW}^{1} / \mathrm{NET}^{2}\right)\end{array}$} \\
\cline { 2 - 4 } & $25 \mathrm{kHz}$ & $50 \mathrm{kHz}$ & $100 \mathrm{kHz}$ \\
\hline $\begin{array}{c}\text { MCS-1 } \\
\left(\pi / 4 \text { QPSK, CR }{ }^{3}=1 / 2\right)\end{array}$ & $38.4 / 15$ & $76.8 / 32$ & $153.6 / 66$ \\
\hline $\begin{array}{c}\text { MCS-3 } \\
(8 \mathrm{PSK}, \mathrm{CR}=3 / 4)\end{array}$ & $57.6 / 35$ & $115.2 / 74$ & $230.4 / 150$ \\
\hline $\begin{array}{c}\mathrm{MCS}-5 \\
(16 \mathrm{QAM}, \mathrm{CR}=3 / 4)\end{array}$ & $76.8 / 47$ & $153.6 / 100$ & $307.3 / 200$ \\
\hline
\end{tabular}

${ }^{1} \mathrm{RAW}$ - raw bit rate, ${ }^{2} \mathrm{NET}-$ net bit rate, ${ }^{3} \mathrm{CR}-$ code rate ${ }^{*}$

\section{PHYSICAL LAYER OF VDE-TER SEGMENT}

The physical layer of the VDE-TER segment is responsible for transmission and reception of the bit data stream, including channel coding, modulation, symbol shaping, as well as signal filtering and synchronization [12].

For each band, the structure of the frame has been defined (Fig. 1). The frame duration is the same for every bandwidth and equals $26.667 \mathrm{~ms}$. On the other hand, the number of symbols transmitted in a single frame varies depending on

\footnotetext{
* The code rate $k / n$ is the ratio of the $k$-bit information sequence and the corresponding $n$-bit code word sequence.
}

the bandwidth: in the case of $25 \mathrm{kHz}$ there are 512 symbols per frame, in the case of $50 \mathrm{kHz}-1024$ symbols, and in the case of $100 \mathrm{kHz}-2048$ symbols.

As we can see, the frame comprises five blocks:

- The initial block is responsible for power detector's activation,

- The training sequence is composed of the bit ' 1 ' concatenated with two orthogonal Barker codes (1+Barker13+Inversed Barker13, so the actual sequence is: 1 1111100110101 0000011001010). The Barker codes have very good autocorrelation properties, which makes the detection of subsequent frames simple and reliable.

- The third block contains the basic information about the MCS and it is comprised of four bits which are encoded using the Hamming $(7,4)$ code. The resulting sequence is 7 bit long.

- The data block carries the encoded user data based on cyclic and correction code.

- A buffer appended to the frame acts as a time reserve to eliminate the influence of transmission delays caused by the varying distance between the transmit and receive stations.

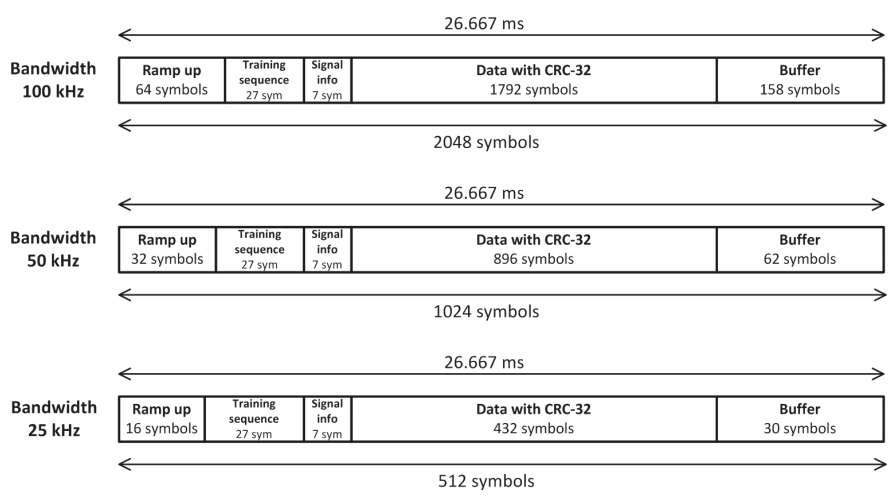

Fig. 1. Single frame structure for the VDE-TER

Figs. 2 and 3 show simplified block diagrams of the VDETER transmitter and receiver, respectively.

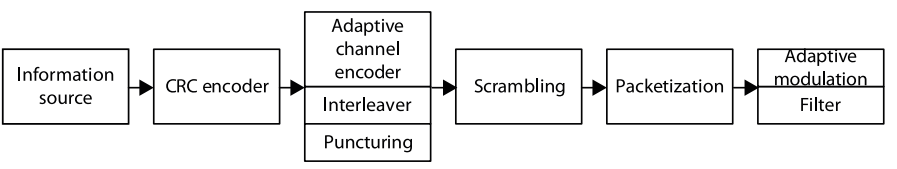

Fig. 2. Simplified VDE-TER transmitter block diagram

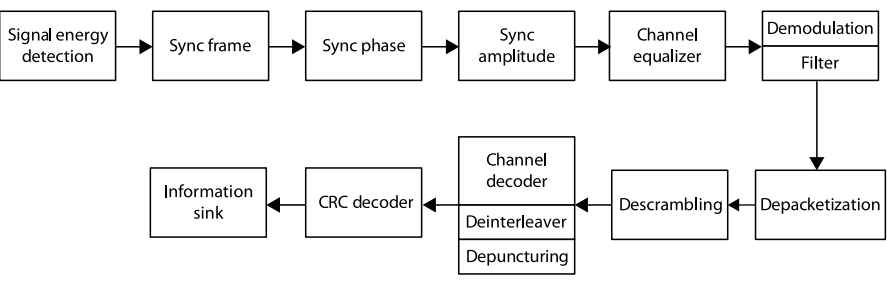

Fig. 3. Simplified VDE-TER receiver block diagram

The first step of the signal transmission process is source data generation. The information sequence prepared in this way is then fed into the CRC 32 cyclic encoder, where the $32 \mathrm{bit}$ 
checksum is appended. In the next stage, channel encoding is performed using a turbocoder. After that, the processed data is fed into the scrambler and randomized. The resulting data sequence is then packetized, modulated and filtered, and finally it is transmitted over the radio channel.

The first step of the reception procedure is signal detection. It is performed using a power detector. The subsequent stages execute time synchronization, phase and frequency synchronization, and amplitude scaling [18]. After that, the coefficients of the channel equalizer's filter are calculated [7]. Next, filtering and demodulation of the received signal is carried out. Then, the bits are fed into the depacketizer block which selects only those bits that belong to a data block. Each received block is descrambled and turbodecoded. The data reconstructed after turbodecoding is validated (using the CRC32 cyclic decoder) and finally the estimation of the source information is produced.

Turbocoding is a method to detect and correct errors. It is the most significant way to ensure high reliability of digital transmission. The turbocoding (based on the 3GPP2 turbocode) in the VDES system is performed using an interleaver, two identical systematic encoders of the convolutional code, and a puncturing block. The same turbocoder is used for each MCS, but the interleavers and puncturing algorithms vary depending on a scheme that is being utilized. Generally, the standard [12] defines nine versions of the interleaver (one for every possible combination of MCS/bandwidth) and two versions of puncturing (either to obtain $\mathrm{CR}=1 / 2$ or $\mathrm{CR}=3 / 4$, see Table 1 ). The turbodecoder, on the other hand, is comprised of two convolutional code decoders, and interleaver and deinterleaver blocks. The decoding process is performed using a soft-decision Viterbi algorithm SOVA (Soft Output Viterbi Algorithm), in which the metrics are calculated using soft decisions produced by two decoders which "support" each other in subsequent turbodecoding iterations [20].

In the VDE-TER system, three modulation schemes have been defined: $\pi / 4$ QPSK, 8PSK, and 16QAM. It is assumed that the preamble block (including training sequence and "signal info" field) and the buffer are always modulated using the $\pi / 4$ QPSK modulation in order to ensure reliable time synchronization (in case of user data, each of the three possible modulation schemes can be used depending on the channel quality). Additionally, to enable soft turbodecoding, each of the analyzed demodulators is equipped with a softoutput decision-making block.

The Root Raised Cosine filter (RRC), with Roll-off factor $=0.3$, is applied both in the modulator and demodulator. It is used to reduce the modulated signal bandwidth and to minimize the intersymbol interference (ISI).

The essential units in the VDE-TER segment are those responsible for synchronization and reception of signals. The receiving process starts in the signal power detector, which checks whether the average received signal power is above the designated threshold. When the receiver detects a signal, it is necessary to determine the position of the transmitted frame. As soon as there are enough samples of the signal in the receiver's memory, the time synchronization algorithm initiates its operation. The received signal is correlated with the known reference signal (this is possible due to the training sequence placed in the frame structure). The training sequence is composed of two Barker codes (see the description above) which have very good autocorrelation properties. Once the frame's position has been determined and the sufficient amount of data accumulated, the signal phase needs to be evaluated. Phase synchronization consists in comparing the received training symbol positions with their reference positions. Based on the known signal constellation, the amplitude of the received signal is also determined.

In the VDE-TER segment, the system self-adjusts to the condition of the radio channel. It is assumed that on the basis of the MER parameter (Modulation Error Ratio [5]), the system adapts itself to the most suitable modulation and coding scheme (when the transmitter obtains the feedback information from the receiver).

The value of this parameter depends on the level and type of noise that interferes with the signal. Generally, the MER should be interpreted as a distance from the total signal fading and it can be calculated using the following equation [3]:

where:

$$
\operatorname{MER}(d B)=10 \log _{10}\left(\frac{P_{\text {signal }}}{P_{\text {error }}}\right)
$$

$\mathrm{P}_{\text {signal }}$ - RMS (Root Mean Square) power of the 'perfect' signal,

$\mathrm{P}_{\text {error }}-\mathrm{RMS}$ power of the very strongly distorted signal.

The implemented simulator allows to enter the MER value at which the system should change its current MCS scheme.

It is of crucial importance to correctly determine which modulation and coding scheme has been used by the transmitter. On the basis of this information, the receiver selects the appropriate demodulator. The MCS information encoded by the Hamming code is placed in the frame field "signal info" and modulated using the $\pi / 4$ QPSK modulation. Consequently, to obtain the information about MCS it is necessary to demodulate the received signal and decode the relevant field in the frame. In order to improve the signalto-noise ratio and to increase the detection probability of the "signal info" field, the following mapping applies to the training and signal information:

- " 1 " is mapped to $\pi / 4$ QPSK symbol $3(1,1)$

- " 0 " is mapped to $\pi / 4$ QPSK symbol $0(0,0)$.

By utilizing various MCS schemes and the measured MER, the system can work adaptively, i.e. it can adapt itself to radio channel conditions, thus maximizing the throughput and transmission quality.

In order to minimize the way in which fadings might affect the transmitted signal, a ZF (Zero Forcing) channel equalizer [14], [15], [16] has been introduced. The task of this module is to determine the filter coefficients on the basis of the synchronization signal and the known reference signal.

This module is necessary to minimize the influence of fadings that exist in the maritime radio channel - especially when a ship is in close proximity to the shore, and signal reflection and heavy attenuation can occur. 
The last stage of signal processing in the receiver is its filtering and demodulation. Three demodulators have been implemented in the VDE-TER software simulator: $\pi / 4$ QPSK, 8PSK, and 16QAM. The decision-making blocks are different for each demodulator. Additionally, for the needs of turbodecoding, each of the analyzed demodulators is equipped with a soft-output decision-making module.

\section{TRANSMISSION CHANNELS}

In the VDE-TER simulator, three transmission channels have been implemented:

- AWGN (Additive White Gaussian Noise) channel,

- Rayleigh channel - Rayleigh fading channel with additive white Gaussian noise,

- Two-path AWGN channel - two-path maritime channel with additive white Gaussian noise and slow fading.

The first two channels are commonly known and frequently utilized in simulations and analyses of radiocommunication systems (see e.g. [19]), so only the third channel will be introduced here in a more detailed way.

\section{MARITIME RADIO CHANNEL}

To facilitate the analysis of a new wireless data transmission system at sea, a novel maritime radio channel model in the VHF band has been defined by IALA. It was observed that in maritime conditions, two channel cases can be distinguished:

- The case where the ship is out at sea, and the multipath propagation is virtually nonexistent (the observations have shown that the delay of the second propagation path on the high seas is a few nanoseconds). Consequently, the only transmission channel in this case is practically an AWGN channel.

- The case where the ship is in the harbor or its vicinity. In this scenario, the multipath effect is significant, and consequently, after investigations, it is recommended. This special scenario should be modelled as a two-tap AWGN channel in which the second path attenuation varies depending on the ship location and the number of reflecting objects in the harbor [6].

In both scenarios mentioned above, it is assumed that due to small speed of ships and other objects nearby, the Doppler offset is small and almost constant. Consequently, the Rayleigh fading effect is virtually nonexistent.

Therefore, three variants of the maritime radio channel have been proposed, with their parameters listed below:

- Delay of the second path in relation to the main path is $10 \mu \mathrm{s}$,

- Attenuation of the second path (second tap) with respect to the first one depends on the severity of the multipath phenomenon and might be equal to: $3 \mathrm{~dB}, 6 \mathrm{~dB}$ or $10 \mathrm{~dB}$,

- Doppler shift is almost constant and does not exceed $5 \mathrm{~Hz}$ at $160 \mathrm{MHz}$.

\section{SIMULATION RESULTS}

The simulation research has been carried out using the software developed by the authors in the $\mathrm{C}++$ language. The transceiver and radio channel blocks have been implemented in software. In each simulation, the number of transmitted frames (sufficient to reliably obtain the BER equal to $10^{-6}$ ) and the appropriate MCS scheme for a given bandwidth were indicated. Phase synchronization, amplitude estimation, and scrambler (descrambler) and turbocoder (turbodecoder) blocks were also included. Furthermore, the simulations were performed for two transmission channels (AWGN and the maritime radio channel).

\section{CHARACTERISTICS OF BER AND BLER FOR VARIOUS MCSS}

The characteristics of bit error rate (BER) vs. $\mathrm{E}_{\mathrm{b}} / \mathrm{N}_{0}$ and block error rate (BLER) vs. $\mathrm{E}_{\mathrm{s}} / \mathrm{N}_{0}$ for MCS-1, MCS3 and MCS-5 in the AWGN channel are presented in Figs. 4, 5 and 6. In each case, the results have been obtained for three channel bandwidths: $25 \mathrm{kHz}, 50 \mathrm{kHz}$, and $100 \mathrm{kHz}$. The bit error rate characteristics have been compared to the theoretical curves for QPSK (MCS-1), 8PSK (MCS-3) and 16QAM (MCS5).

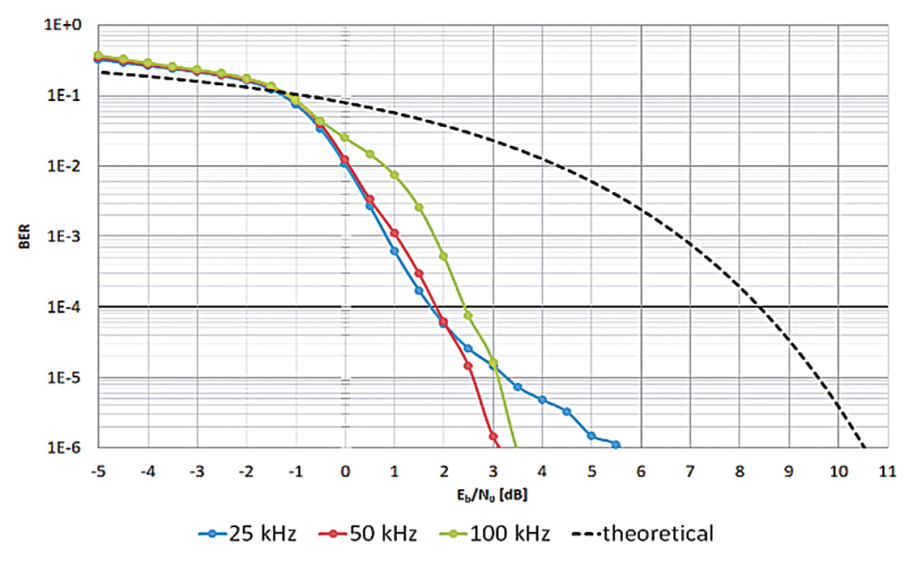

Fig. 4. BER vs. $E_{b} / N_{0}$ for MCS-1 in the AWGN channel

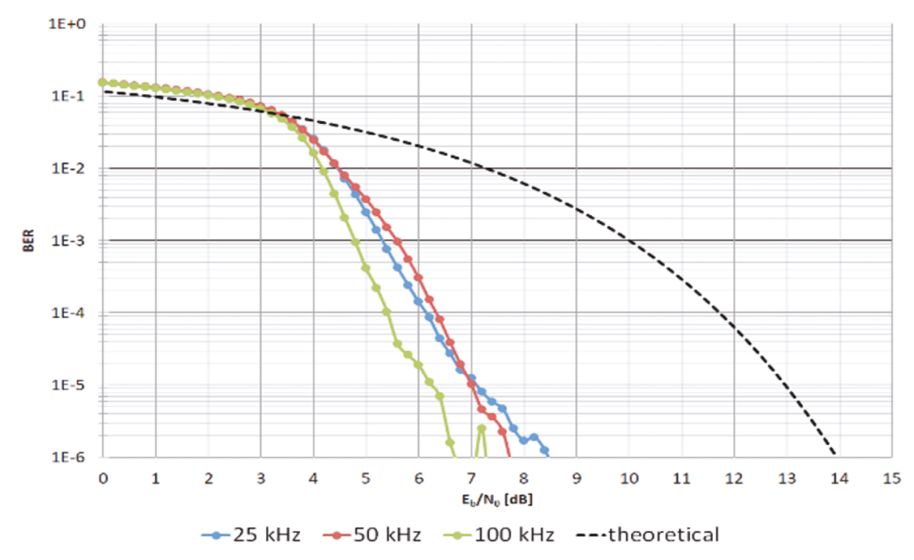

Fig. 5. BER vs. $E_{b} / N_{0}$ for MCS-3 in the AWGN channel 


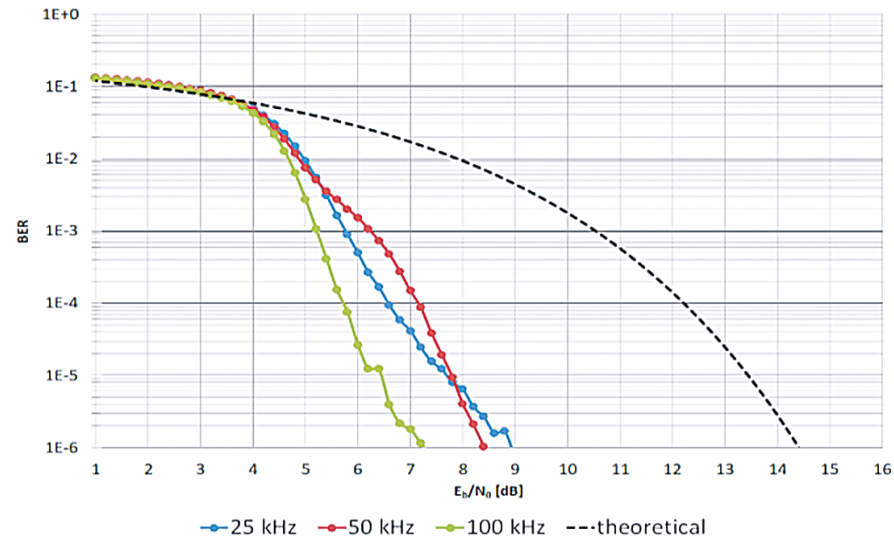

Fig. 6. BER vs. $E_{b} / N_{0}$ for MCS-5 in the AWGN channel

Table 2 collates coding gains observed for each of the analyzed MCS schemes. The gain values have been calculated with respect to the theoretical BER curves, for BER value of $10^{-6}$.

Tab. 2. Coding gains for $B E R=10^{-6}$

\begin{tabular}{|c|c|c|c|}
\hline \multirow{2}{*}{$\begin{array}{c}\text { Modulation and coding } \\
\text { scheme (MCS) }\end{array}$} & \multicolumn{3}{|c|}{ Coding gain [dB] } \\
\cline { 2 - 4 } & $25 \mathrm{kHz}$ & $50 \mathrm{kHz}$ & $100 \mathrm{kHz}$ \\
\hline MCS-1 & 5 & 7 & 7.5 \\
\hline MCS-3 & 5.5 & 6 & 6 \\
\hline MCS-5 & 7.5 & 7.5 & 7.3 \\
\hline
\end{tabular}

As we can see, the largest coding gain can generally be achieved for the $100 \mathrm{kHz}$ bandwidth, because in this case the data blocks are the longest, which improves the turbodecoding quality. On the other hand, the $100 \mathrm{kHz}$ bandwidth requires highly reliable time synchronization, as one symbol contains 4 signal samples. Consequently, any synchronization error (e.g. shift by one sample on the time scale) might result in a transmission error. This issue is much less significant in the $50 \mathrm{kHz}$ and $25 \mathrm{kHz}$ bandwidths, because in these cases one symbol is comprised of 8 and 16 samples, respectively.

The block error rate characteristics indicate the $\mathrm{E}_{\mathrm{s}} / \mathrm{N}_{0}$ values at which at least one block of data on the average can be received correctly. As we can see in Figs. 7-9, in the case of MCS-1 not even one block can be sent successfully if $\mathrm{E}_{\mathrm{s}} / \mathrm{N}_{0}$ is less than $2.5 \mathrm{~dB}$. In the cases of MCS-3 and MCS-5, the respective $\mathrm{E}_{\mathrm{s}} / \mathrm{N}_{0}$ threshold values are $9 \mathrm{~dB}$ and $11 \mathrm{~dB}$.

Table 3 presents the simulation results obtained for three radio channel models (AWGN and two versions of the maritime radio channel) and for selected $\mathrm{E}_{\mathrm{b}} / \mathrm{N}_{0}$ values. These results indicate that the first version of the maritime channel (delay $=30 \mu \mathrm{s}$, attenuation $=20 \mathrm{~dB}$ ) has slightly worse BER characteristics compared to the AWGN channel. On the other hand, the utilization of the other maritime channel (delay $=10 \mu \mathrm{s}$, attenuation $=3 \mathrm{~dB}$ ) results in substantial degradation of the reception quality (BER increases by several orders of magnitude).

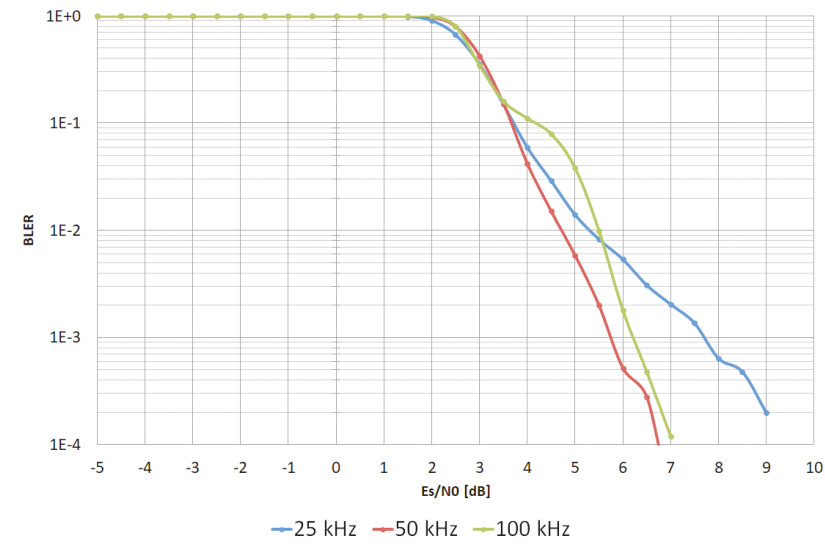

Fig. 7. BLER vs. $E_{s} / N_{0}$ for MCS-1 in the AWGN channel

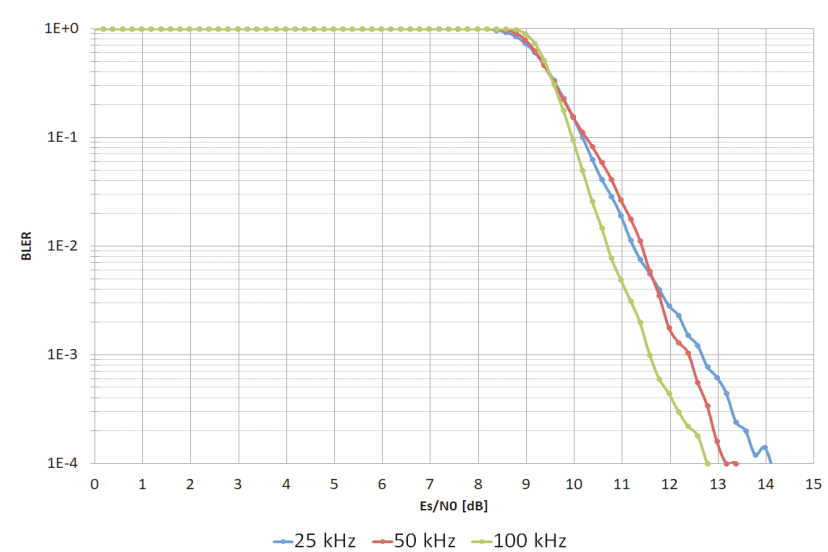

Fig. 8. BLER vs. $E_{s} / N_{0}$ for MCS-3 in the AWGN channel

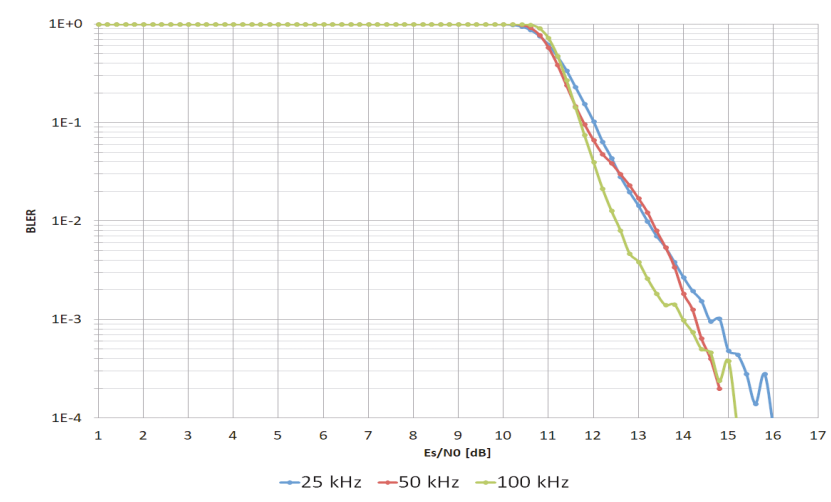

Fig. 9. BLER vs. $E_{s} / N_{0}$ for MCS-5 in the AWGN channel

Tab. 3. BER for three different radio channels (without equalizer)

\begin{tabular}{|c|c|c|c|c|}
\hline & \multirow[b]{2}{*}{ Bandwidth } & \multirow{2}{*}{$\begin{array}{l}\text { AWGN } \\
\text { channel }\end{array}$} & \multicolumn{2}{|c|}{ Maritime channel } \\
\hline & & & $\begin{array}{c}\text { delay }=30 \mu \mathrm{s} \\
\text { att. }=20 \mathrm{~dB}\end{array}$ & $\begin{array}{c}\text { delay }=10 \mu \mathrm{s} \\
\text { att. }=3 \mathrm{~dB}\end{array}$ \\
\hline \multirow{3}{*}{ MCS-1 } & $\begin{array}{c}25 \mathrm{kHz} \\
\left(\mathrm{E}_{\mathrm{b}} / \mathrm{N}_{0}=5,5 \mathrm{~dB}\right)\end{array}$ & $1,1 \mathrm{E}-6$ & $6,0 \mathrm{E}-7$ & $8,8 \mathrm{E}-7$ \\
\hline & $\begin{array}{c}50 \mathrm{kHz} \\
\left(\mathrm{E}_{\mathrm{b}} / \mathrm{N}_{0}=3 \mathrm{~dB}\right)\end{array}$ & 1,4 E-6 & $2,5 \mathrm{E}-6$ & $3,5 \mathrm{E}-5$ \\
\hline & $\begin{array}{c}100 \mathrm{kHz} \\
\left(\mathrm{E}_{\mathrm{b}} / \mathrm{N}_{0}=3,5 \mathrm{~dB}\right)\end{array}$ & 8,5 E-7 & $1,1 \mathrm{E}-5$ & $1,1 \mathrm{E}-2$ \\
\hline
\end{tabular}




\begin{tabular}{|c|c|c|c|c|}
\hline & \multirow[b]{2}{*}{ Bandwidth } & \multirow{2}{*}{$\begin{array}{l}\text { AWGN } \\
\text { channel }\end{array}$} & \multicolumn{2}{|c|}{ Maritime channel } \\
\hline & & & $\begin{array}{l}\text { delay }=30 \mu \mathrm{s} \\
\text { att. }=20 \mathrm{~dB}\end{array}$ & $\begin{array}{c}\text { delay }=10 \mu \mathrm{s} \\
\text { att. }=3 \mathrm{~dB}\end{array}$ \\
\hline \multirow{3}{*}{ MCS-3 } & $\begin{array}{c}25 \mathrm{kHz} \\
\left(\mathrm{E}_{\mathrm{b}} / \mathrm{N}_{0}=8,4 \mathrm{~dB}\right)\end{array}$ & $1,3 \mathrm{E}-6$ & $1,2 \mathrm{E}-6$ & $3,1 \mathrm{E}-6$ \\
\hline & $\begin{array}{c}50 \mathrm{kHz} \\
\left(\mathrm{E}_{\mathrm{b}} / \mathrm{N}_{0}=7,8 \mathrm{~dB}\right)\end{array}$ & $6,7 \mathrm{E}-7$ & $6,7 \mathrm{E}-7$ & $1,1 \mathrm{E}-3$ \\
\hline & $\begin{array}{c}100 \mathrm{kHz} \\
\left(\mathrm{E}_{\mathrm{b}} / \mathrm{N}_{0}=6,8 \mathrm{~dB}\right) \\
\end{array}$ & $6,7 \mathrm{E}-7$ & $1,4 \mathrm{E}-6$ & $1,5 \mathrm{E}-1$ \\
\hline \multirow{3}{*}{ MCS-5 } & $\begin{array}{c}25 \mathrm{kHz} \\
\left(\mathrm{E}_{\mathrm{b}} / \mathrm{N}_{0}=9 \mathrm{~dB}\right) \\
\end{array}$ & 7,7 E-7 & $1,1 \mathrm{E}-6$ & 6,9 E-6 \\
\hline & $\begin{array}{c}50 \mathrm{kHz} \\
\left(\mathrm{E}_{\mathrm{b}} / \mathrm{N}_{0}=8,4 \mathrm{~dB}\right)\end{array}$ & $1,0 \mathrm{E}-6$ & $6,7 \mathrm{E}-7$ & $2,3 \mathrm{E}-2$ \\
\hline & $\begin{array}{c}100 \mathrm{kHz} \\
\left(\mathrm{E}_{\mathrm{b}} / \mathrm{N}_{0}=7 \mathrm{~dB}\right)\end{array}$ & $1,8 \mathrm{E}-6$ & $2,2 \mathrm{E}-6$ & $1,7 \mathrm{E}-1$ \\
\hline
\end{tabular}

\section{THE INFLUENCE OF SYNCHRONIZATION ERROR ON TRANSMISSION QUALITY}

Figure 10 shows the characteristics of the following three parameters as functions of $\mathrm{E}_{\mathrm{s}} / \mathrm{N}_{0}$ :

- Time synchronization error rate (denoted as 'sync time error' in the figure),

- Error of "signal info" field reception (denoted as 'signal info error') - the "signal info" field carries the information about the current MCS scheme, so the erroneous reception of this field may result in incorrect signal demodulation,

- Block error rate (BLER).

These curves have been drawn for MCS-1.

Figure 10 illustrates how the synchronization error and errors of "signal info" field reception affect the transmission quality.

In the perfect scenario, there shouldn't be any sync and signal info errors when $\mathrm{E}_{\mathrm{s}} / \mathrm{N}_{0}$ is large enough to send at least one block correctly (i.e. when BLER is less than 1). As was mentioned in the previous subsection, this threshold value of $\mathrm{E}_{\mathrm{s}} / \mathrm{N}_{0}$ is approx. $2.5 \mathrm{~dB}$ (for MCS-1).

In the case of MCS-1, this perfect scenario is not achieved, but the results are still satisfactory. As we can observe, at $\mathrm{E}_{\mathrm{s}} / \mathrm{N}_{0}=2.5 \mathrm{~dB}$, the signal info error is less than $10^{-2}$ and the sync error is less than $10^{-4}$. Practically, it is assumed that the transmission is correct when BLER is less than $10^{-1}$, and this condition is satisfied in the discussed figure.

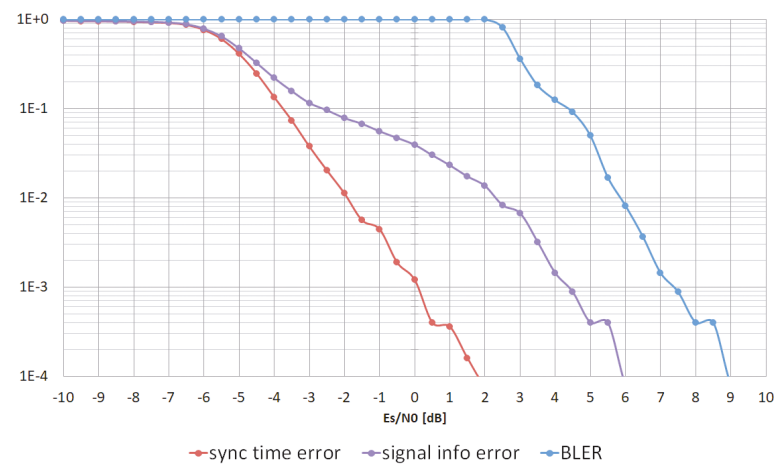

Fig. 10. Synchronization error rate, "signal info" field reception error, and BLER for MCS-1

\section{THE EFFICIENCY OF THE ZERO-FORCING CHANNEL EQUALIZER}

Figure 11 shows the 8PSK modulation constellation (MCS-3) for the AWGN channel (a), maritime radio channel without equalizer (b), and maritime radio channel with equalizer (c).

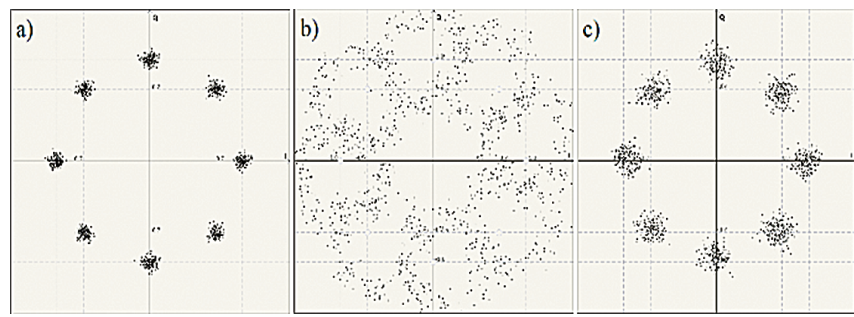

Fig. 11. MCS-3 signal constellation for: a) AWGN channel without equalizer, b) maritime channel without equalizer, c) maritime channel with equalizer

This figure mainly demonstrates the efficiency of the zeroforcing channel equalizer when used in the VDES system. In the discussed scenario, the ZF equalizer is used in the presence of selective frequency fadings. New characteristics of the filter are calculated for every subsequent training sequence, i.e. for every frame. If the equalization is not used, the fading and intersymbol interference (ISI) strongly affect the transmitted signal and make correct transmission almost impossible (see fig. 11b). Obviously, this issue is becoming more serious when higher-order modulations are utilized. On the other hand, the signal constellations show that the influence of ISI is substantially reduced when the equalizer is used (theoretically, the ZF equalizer is perfect, i.e. removes all ISI in a fully noiseless channel). At the same time, we might also observe that using the equalizer will result in noise amplification; it is a drawback of this solution, but it is consistent with the general theory of the zero-forcing equalizer.

It should be mentioned here that for the purpose of the discussed simulations, a slightly modified version of the ZF equalizer was implemented, which assumed adding a type of a limiter. Theoretically, in the presence of deep fading, the filer's coefficients for some frequencies would be enormous and the filter characteristics would approach infinity, which would be impossible to implement. To avoid such situations, the filter coefficients whose values were above the threshold were simply limited ("truncated") by the algorithm.

\section{THE INFLUENCE OF CHANNEL INFORMATION DELAY ON THE ACHIEVABLE BIT RATES}

Figures 12-14 illustrate the influence of the auxiliary information about the channel state on the transmission rate. Since the VDES system utilizes adaptive modulation, the timely information about the current state of the radio channel is of paramount importance and the lack of such data significantly degrades the efficiency and performance of the system.

The characteristics in Figs. 12-14 represent the bit rate vs. $\mathrm{E}_{\mathrm{b}} / \mathrm{N}_{0}$ in the AWGN channel and have been obtained for three bandwidths: $25 \mathrm{kHz}, 50 \mathrm{kHz}$ and $100 \mathrm{kHz}$. Two cases of channel information delay have been considered: no delay (red bars) and 3 frames delay (blue bars). 
Obviously, as the $\mathrm{E}_{\mathrm{b}} / \mathrm{N}_{0}$ increases, so does the bit rate, which is mainly due to the adaptive modulation and coding algorithms. The better the channel quality, the better the MCS and, consequently, the higher the bit rate.

At the same time, we can observe that the channel state info delay negatively affects the bit rate for $\mathrm{E}_{\mathrm{b}} / \mathrm{N}_{0}$ from $5 \mathrm{~dB}$ to $9 \mathrm{~dB}$. For other $\mathrm{E}_{\mathrm{b}} / \mathrm{N}_{0}$ values, the resulting bit rates are almost identical and do not seem to be affected by the discussed delay. The above observation is true for every analyzed bandwidth.

These results have shown that in the future stages of VDES development, a new MCS scheme should be introduced to increase the achievable bit rates.

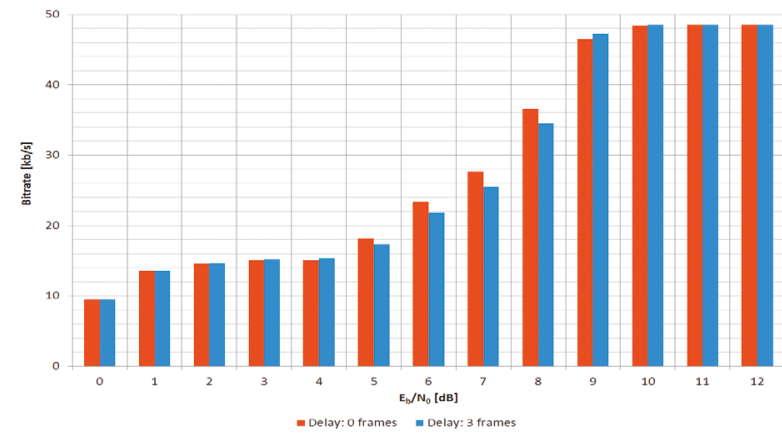

Fig. 12. Bit rate vs. $E_{b} / N_{0}$ for $25 \mathrm{kHz}$ AWGN channel

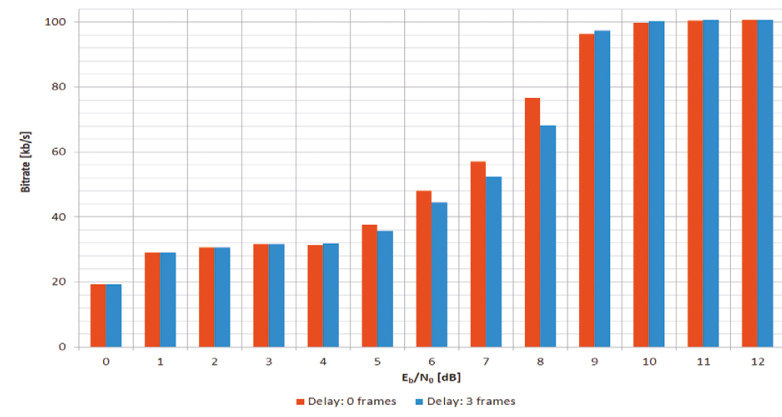

Fig. 13. Bit rate vs. $E_{b} / N_{0}$ for $50 \mathrm{kHz} A W G N$ channel

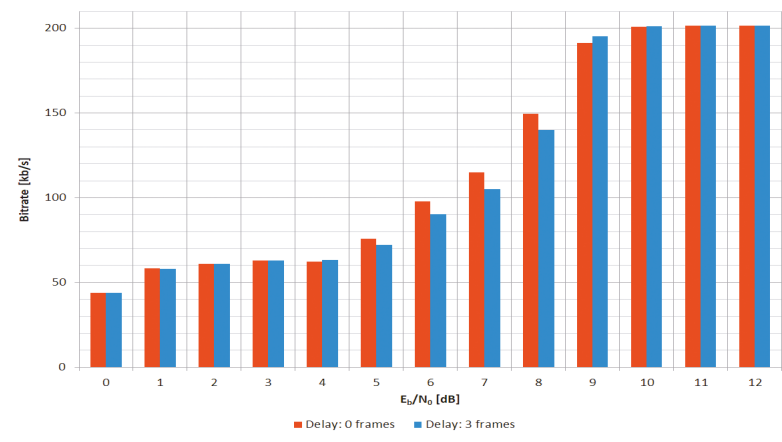

Fig. 14. Bit rate vs. $E_{b} / N_{0}$ for $100 \mathrm{kHz} A W G N$ channel

\section{MEASUREMENT RESULTS}

In the period between 23 March 2017 and 6 April 2017, a measurement campaign of the VDES physical layer was carried out as part of the EfficienSea 2.0 project. As a result, roughly 6 terabytes of measurement data were collected and subsequently subjected to further, more thorough, analysis.
The main goal of this analysis and processing was to obtain the bit error rate characteristics that could serve as a basis for some conclusions regarding the performance of the system. Most importantly, it allowed to compare the results obtained during the simulation analysis with the actual measurement data. For the purpose of this measurement campaign, the receiver part of the VDES simulator was utilized [1]. Additionally, a software interface has been developed which allowed to process data samples collected during the measurements at sea.

Figure 15 presents the general idea of the measurements. As it can be seen, during the campaign, one VDES transmitter and two VDES receivers were utilized. The transmitter was located in the Gedser Havn port (Denmark), whereas one of the receivers was aboard the Scandlines Ferry M/F Berlin, and the other one was aboard the Scandlines Ferry M/F Copenhagen. To make the measurements as realistic as possible, the installation aboard those vessels took advantage of the existing VHF installation. To verify how antenna height affects the propagation, the antennas on the two vessels were installed at different heights (M/F Berlin: $18 \mathrm{~m}$ and M/F Copenhagen: $23 \mathrm{~m}$ ). The entire route length from Gedser to Rostock was about $48 \mathrm{~km}$ (one-way). It should be mentioned that when the ferries were at the maximum possible distance away from the transmitter, they were partly in the inland area, which made the measurement results even more valuable, since in that case the level of interference was higher and the propagation conditions - much tougher.

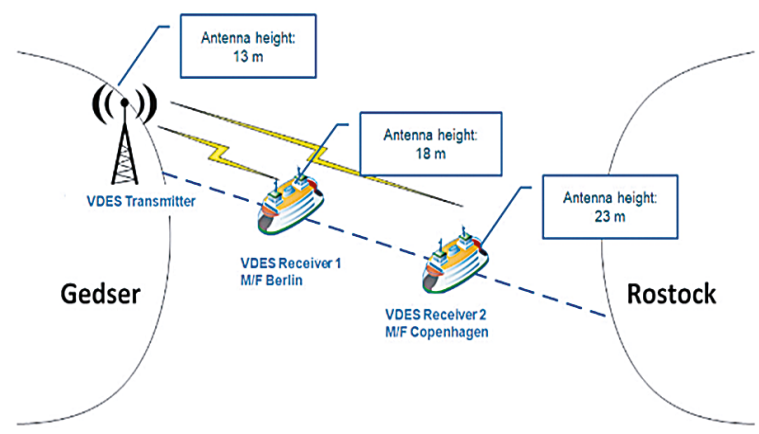

Fig. 15. General concept of the measurement campaign at sea

The technical parameters of the transmitter and two receivers used during the campaign are listed below.

Technical parameters of the transmitter:

- Height of the antenna above ground: $13 \mathrm{~m}$,

- Height of the ground in the transmitter's location: $2 \mathrm{~m}$ a.s.l.,

- Frequency band: $160 \mathrm{MHz}$,

- Distance between the antenna's location and the shore: $0 \mathrm{~m}$ at the ferry landing bridge,

- Transmitter power: 6.3 W ( $\pi / 4$-QPSK) or $4 \mathrm{~W}$ (16QAM),

- Antenna gain: $3 \mathrm{dBi}$,

- Antenna cable length and type: 12 m, RG 214.

Technical parameters of the receivers:

- Height of the antenna: M/F Berlin: $18 \mathrm{~m}, \mathrm{M} / \mathrm{F}$ Copenhagen: $23 \mathrm{~m}$, 
- Location description: one ship: top of the wheel house, the other one: top of the radar mast,

- Receiver sensitivity: $-110 \mathrm{dBm}(\pi / 4-\mathrm{QPSK})$ or $-96 \mathrm{dBm}$ (16-QAM),

- Antenna gain: $3 \mathrm{dBi}$,

- Antenna cable length and type: 17 m, RG 214.

A hardware platform used during the measurements was a proprietary solution comprised of a digital signal processor and a FPGA module on which the transmission and receive channels were implemented. A digital-to-analog converter and an analog-to-digital converter were also part of the setup, as well as the DR 200 broadband power amplifier (version D) made by Prana. The bits were processed separately in the I and $\mathrm{Q}$ channels (that includes filtering and mixing). The receive data rate of the FPGA was $19.66 \mathrm{Mbps}$, and the sampling rate was $4.9152 \mathrm{MHz}$.

\section{DATA SEQUENCE UTILIZED DURING THE MEASUREMENT CAMPAIGN}

During the measurement campaign, a predefined data sequence (lasting 20 seconds in total) was employed. That sequence was transmitted in a defined order and that order was strictly followed throughout the entire campaign. The transmitter located in the Gedser port transmitted the data in the following sequence:

- First, the data was transmitted in the $25 \mathrm{kHz}$ bandwidth in the following order: MCS-1, MCS-3, MCS-5,

- Then, the data was transmitted in the $50 \mathrm{kHz}$ bandwidth in the following order: MCS-1, MCS-3, MCS-5,

- After that, the data was transmitted in the $100 \mathrm{kHz}$ bandwidth in the following order: MCS1, MCS-3, MCS-5,

- No transmission took place during the periods of switching between different bandwidths. During those periods the slots could be occupied by noise or other interference signals.

The transmitted data sequence spans across a number of time slots assigned to specific modulation schemes. Table 4 contains the information regarding the number of slots available for a given MCS. Additionally, a total duration of the time slot series which depends on the utilized modulation scheme is included.

As it can be seen, it takes exactly 20 seconds to transmit the entire sequence. At this point it should be mentioned that one measurement file covers 60 seconds of data (i.e. three transmitted sequences - a total of 2250 time slots).

During the time slots when no data is sent, the transition from one available bandwidth to another is being performed. That approach has been chosen to facilitate smooth filter passband change. Eight slots with noise located at the end of the transmitted sequence allowed for the measurements of noise power and distortion in the receiver.

It should be noted that it was intentional to assign different numbers of slots to different MCSs. The respective number of slots was selected in such a way that the number of user bits was similar for each case (i.e. for each MCS and bandwidth), as discussed in the next subsection. Consequently, the resulting BER characteristics could be reliably compared to one another.

Tab. 4. Number of time slots and their total duration in the transmitted data sequence

\begin{tabular}{|c|c|c|c|c|}
\hline & & $\begin{array}{c}25 \\
\mathrm{kHz}\end{array}$ & $\begin{array}{c}50 \\
\mathrm{kHz}\end{array}$ & $\begin{array}{l}100 \\
\mathrm{kHz}\end{array}$ \\
\hline \multirow{2}{*}{ MCS-1 } & Slots & 250 & 116 & 57 \\
\hline & duration [s] & 6.67 & 3.09 & 1.52 \\
\hline \multirow{2}{*}{ MCS-3 } & Slots & 106 & 50 & 25 \\
\hline & duration $[s]$ & 2.83 & 1.33 & 0.67 \\
\hline \multirow{2}{*}{ MCS-5 } & Slots & 79 & 38 & 19 \\
\hline & duration $[s]$ & 2.11 & 1.01 & 0.51 \\
\hline \multirow{2}{*}{$\frac{\text { NO }}{\text { TRANSMISSION }}$} & Slots & \multicolumn{3}{|c|}{10} \\
\hline & duration [s] & \multicolumn{3}{|c|}{0.27} \\
\hline \multirow{2}{*}{ TOTAL } & Slots & \multicolumn{3}{|c|}{750} \\
\hline & duration $[s]$ & \multicolumn{3}{|c|}{20} \\
\hline
\end{tabular}

\section{ATTENUATION IN THE RECORDED DATA}

The data format used during the measurement campaign contained the information about time periods when additional attenuation was inserted into the analog receiver's path and the receiver did not operate at its maximum sensitivity. Generally, this occurred in two situations:

- The first situation is caused by the VDES signal itself and in the case of the campaign discussed in this document, it occurred when the measurements were conducted close to Gedser (approx. at the distance of 2-3 km),

- The second situation was related to the blocking typically caused by the ships' VHF equipment (AIS, VHF radio).

In Figure 16, the attenuation activity periods are shown as a function of ship's position. It illustrates - for the specific file number - the data percent for which the attenuation was actually active.

Both Fig. 16 and other obtained results confirm that the attenuation was mainly active when the ship was in the vicinity of the shore.

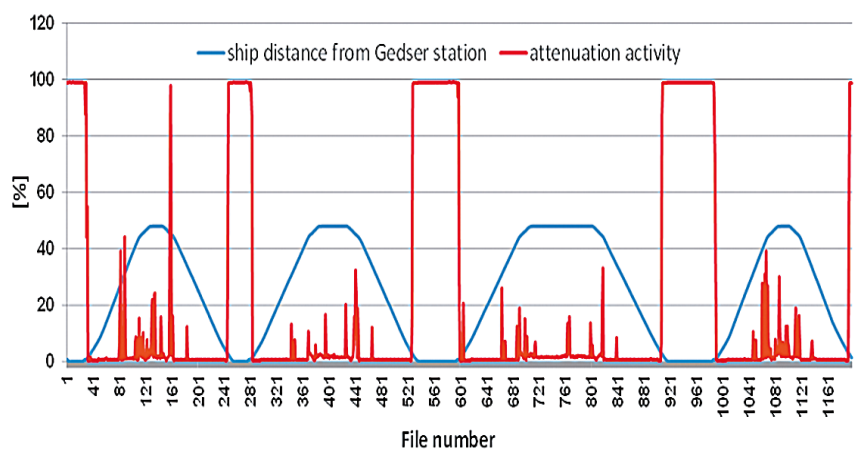

Fig. 16. Attenuation activity vs. file number (ship's distance from Gedser station is also indicated) 


\section{METHOD TO CALCULATE SIGNAL-TO-NOISE RATIO}

In order to obtain correct bit error rate characteristics, it is first necessary to derive the signal-to-noise ratio (usually referred to as SNR) for the given system. This parameter indicates the ratio of the useful signal power to the noise power in the given bandwidth. The expressions (2) and (3) indicate how to calculate SNR and Es/N0 (i.e. the ratio of the energy per symbol to noise power spectrum density).

$$
\begin{gathered}
\frac{S}{N}=\frac{R_{S} \cdot E_{S}}{B_{N} \cdot N_{0}}=\frac{R_{S} \cdot E_{S}}{\frac{F_{S} \cdot N_{0}}{2}}=\frac{2 \cdot R_{S} \cdot E_{S}}{F_{S} \cdot N_{0}} \\
\frac{E_{S}}{N_{0}}=\frac{S \cdot F_{S}}{N \cdot 2 \cdot R_{S}}
\end{gathered}
$$

The symbols used in formulas (2) - (3) represent: $\mathrm{S}$ signal power, $\mathrm{N}$ - noise power, $\mathrm{R}_{\mathrm{s}}-$ symbol rate, $\mathrm{B}_{\mathrm{N}}$ - noise bandwidth, $\mathrm{F}_{\mathrm{s}}$ - sampling frequency, $\mathrm{E}_{\mathrm{s}}$ - energy per symbol, $\mathrm{N}_{0}$ - noise power spectrum density $\left(\mathrm{N}_{0}=\mathrm{kTB}\right.$, where $\mathrm{k}$ is the Boltzmann constant, $\mathrm{T}$ is the absolute temperature of the load, and B is the measurement bandwidth).

It should be noted that the preamble (see Fig. 17) is not used in the $(\mathrm{S}+\mathrm{N})$ calculations according to formulas (2) - (3). It is due to the fact that the preamble is always modulated using the same modulation scheme, i.e. $\pi / 4$-QPSK.

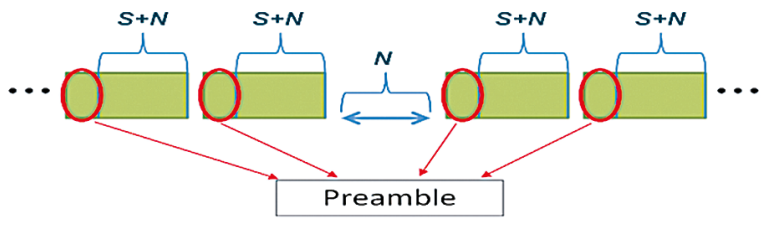

Fig. 17. Illustration of considerations for signal power to noise power determination

The symbol rates for various bandwidths which are necessary to be employed in the formulas considered above are presented in Table 5 [12].

\section{THE ACHIEVABLE RANGE OF THE VDES SYSTEM}

Depending on the modulation scheme and bandwidth, different ranges of the VDES system were recorded. In this case, the term "range" indicates a maximum distance at which the data frame could still be correctly received. The assumed BER threshold value is $10^{-5}$.

The theoretical ranges of the VDES system were calculated using the ITU-R P.1546-5 [11] propagation model. These calculations have been made for two cases:

- Most robust scenario (best case): $\pi / 4$-QPSK modulation and bandwidth of $25 \mathrm{kHz}$,

- Highest throughput scenario (worst case): 16QAM modulation and bandwidth of $100 \mathrm{kHz}$.

Figure 18 presents the maximum and minimum useful ranges for antenna height of $18 \mathrm{~m}$.

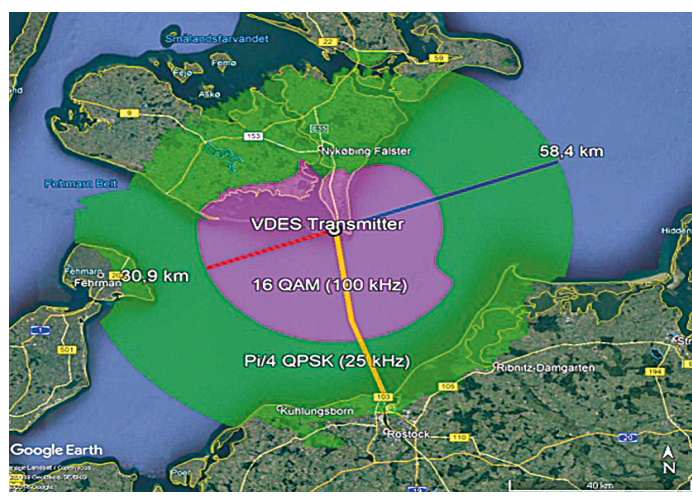

Fig. 18. Maximum and minimum useful range for antenna height of $18 \mathrm{~m}$

The theoretical calculations carried out on the basis of the ITU-R P.1546-5 propagation model indicate that the maximum useful range for the VDES system is about $62.9 \mathrm{~km}$. Such a range is achievable for data reception using the $\pi / 4$-QPSK modulation scheme, the $25 \mathrm{kHz}$ bandwidth, and the receiving antenna height of $23 \mathrm{~m}$. On the other hand, the shortest useful range has been determined for the 16-QAM modulation scheme and the bandwidth of $100 \mathrm{kHz}$. Assuming such a configuration, the achievable range did not exceed $30.9 \mathrm{~km}$ for the receiving antenna height of $18 \mathrm{~m}$.

The measurements confirmed the results of the theoretical analysis realized earlier: the longest range was observed for the $\pi / 4$-QPSK modulation scheme and the bandwidth of $25 \mathrm{kHz}$, whereas for the 16-QAM modulation and the bandwidth of $100 \mathrm{kHz}$, the achievable range was the shortest. Greater antenna height ( $23 \mathrm{~m}$ as opposed to $18 \mathrm{~m}$ ) resulted in the range increase by approx. $3 \mathrm{~km}$.

Figures 19 and 20 present the useful VDES ranges. The curves were drawn for two different antenna heights and for two different modulation schemes and bandwidths, represented here by the respective MCS.

In both figures, the line-of-sight distance is presented as the black dotted line. To calculate this parameter, it was first necessary to obtain the radio horizon distance, separately for the transmitter and the receiver. To do so, the following formula [2] was used:

$$
d_{\text {horizon }}[k m] \approx 4.12 \cdot \sqrt{h}
$$

where $h$ is the transmitter/receiver antenna height (in [m]), respectively.

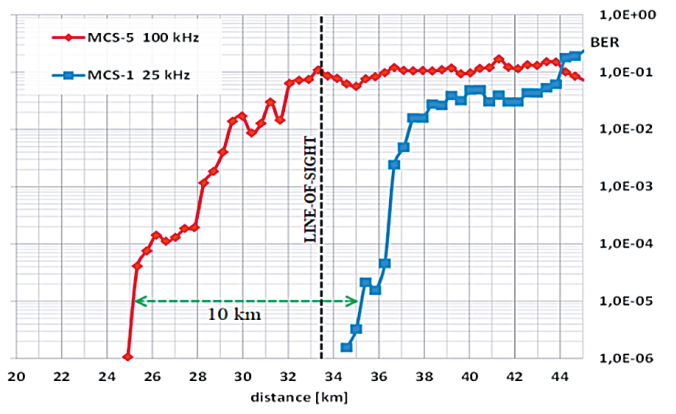

Fig. 19. Useful VDES ranges for MCS-1 and MCS-5 at antenna height of $18 \mathrm{~m}$ 


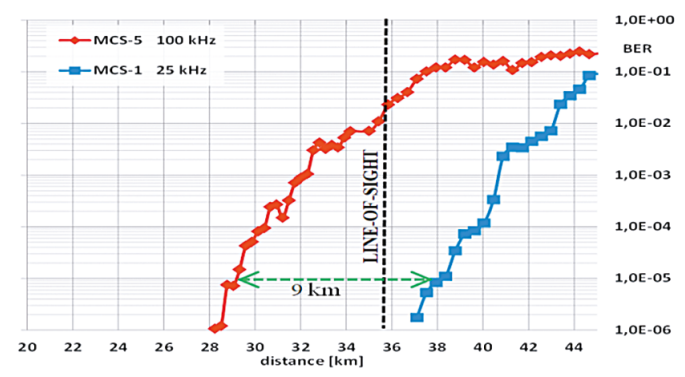

Fig. 20. Useful VDES ranges for MCS-1 and MCS-5 at antenna height of $23 \mathrm{~m}$

Finally, the line-of-sight distance was calculated as the sum of radio horizon distances for the transmitter and the receiver:

$$
\begin{gathered}
d_{\text {LOS }}=d_{\text {horizon }_{\text {transmitter }}}+d_{\text {horizon }_{\text {receiver }}}= \\
=4.12 \cdot\left(\sqrt{h_{\text {transmitter }}}+\sqrt{h_{\text {receiver }}}\right)
\end{gathered}
$$

For the case shown in Fig. 19, where $h_{\text {transmitter }}=15 \mathrm{~m}$ and $h_{\text {receiver }}=18 \mathrm{~m}, d_{\text {LOS }} \approx 33.44 \mathrm{~km}$, while for that shown in Fig. 20 , where $h_{\text {transmitter }}=15 \mathrm{~m}$ and $h_{\text {receiver }}=23 \mathrm{~m}, d_{\text {LOS }} \approx 35.72 \mathrm{~km}$.

It can be observed that in the cases of LOS (line-of-sight) scenarios, the measured useful ranges were generally consistent with the theoretical results obtained using the ITU-R P.1546-5 propagation model [11]. In LOS, the range of the system is mainly limited by the receiver's sensitivity and the AWGN noise.

For the NLOS scenarios (i.e. after crossing the radio horizon line), the consistency between the theoretical and measured data was no longer observed. The ITU-R P.1546-5 model only covers the attenuation increase due to crossing the radio horizon, but it does not include other significant effects that occur in the NLOS situation. The most important factor that limits the actual range in NLOS is signal fading, and this particular phenomenon cannot be analyzed using the ITU-R P.1546 model.

\section{BIT ERROR RATE CHARACTERISTICS OF THE SYSTEM}

The results obtained in the VDES receiver's simulator included bit error rate (BER) characteristics. To calculate them, it was necessary to divide the number of erroneous bits (those with an incorrect checksum or those that could not be decoded due to synchronization errors) by the total number of all transmitted bits.

Such an approach allowed to analyze how the bit error rate actually depends on the signal power for various scenarios, including three modulation schemes and three bandwidths. Table 6 collates the values of $\mathrm{E}_{\mathrm{s}} / \mathrm{N}_{0}$ for which $\mathrm{BER}=10^{-6}$.

Figs. 21 and 22 show the selected bit error rate characteristics of the VDES system.

The analysis of these characteristics shows that the smallest value of $\mathrm{E}_{\mathrm{s}} / \mathrm{N}_{0}$ for $\mathrm{BER}=10-6$ is achieved for the $\pi / 4$-QPSK modulation and the bandwidth of $25 \mathrm{kHz}$, with the antenna height of $23 \mathrm{~m}$. Generally, narrower bandwidths resulted in better bit error rate characteristics. For higher modulation and coding schemes (e.g. MCS-5), substantial degradation
Tab. 6. The values of $E / N_{0}[d B]$ for $B E R=10^{-6}$

\begin{tabular}{|c|c|c|c|c|c|c|}
\hline & \multicolumn{3}{|c|}{ Antenna height $=18 \mathrm{~m}$} & \multicolumn{3}{c|}{ Antenna height=23 m } \\
\cline { 2 - 7 } & MCS-1 & MCS-3 & MCS-5 & MCS-1 & MCS-3 & MCS-5 \\
\hline $\begin{array}{c}25 \\
\mathrm{kHz}\end{array}$ & 6.25 & 10 & 13.25 & 5.5 & 8.75 & 11.75 \\
\hline $\begin{array}{c}50 \\
\mathrm{kHz}\end{array}$ & 8.75 & 11.75 & 14 & 7.75 & 10.5 & 13.5 \\
\hline $\begin{array}{c}100 \\
\mathrm{kHz}\end{array}$ & 10.5 & 13.5 & 15 & 9.25 & 11.75 & 14 \\
\hline
\end{tabular}

of the BER can be observed. This is caused by the fact that higher order modulations (like 16-QAM) - while providing higher throughput and allowing for more efficient use of the spectrum - are much more vulnerable to any interference or distortions than the basic modulation schemes (QPSK).

\section{CONCLUSIONS}

The VHF Data Exchange System is one of the key solutions that will influence the development of maritime communications. According to IMO or IALA assumptions [8], VDES will be one of the essential "platforms" for future e-navigation services and applications, and as such it will also be a crucial system from the safety of navigation point of view.

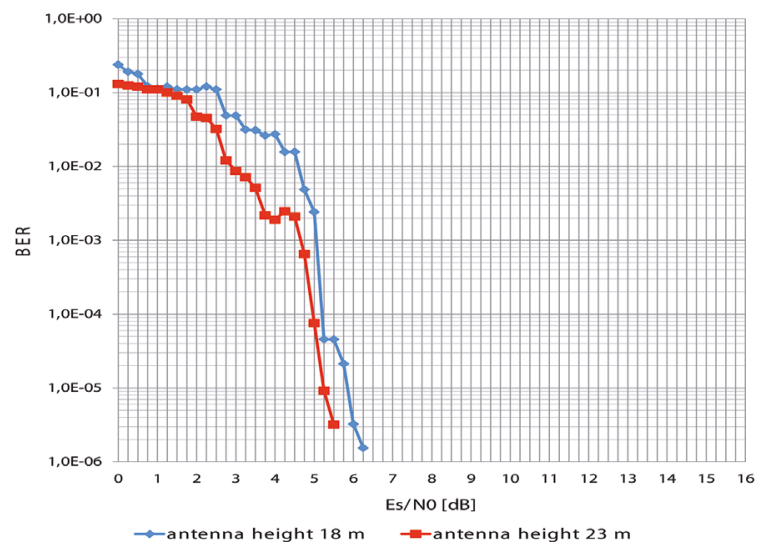

Fig. 21. BER vs. Es $/ N_{0}$ for MCS-1 and bandwidth $25 \mathrm{kHz}$

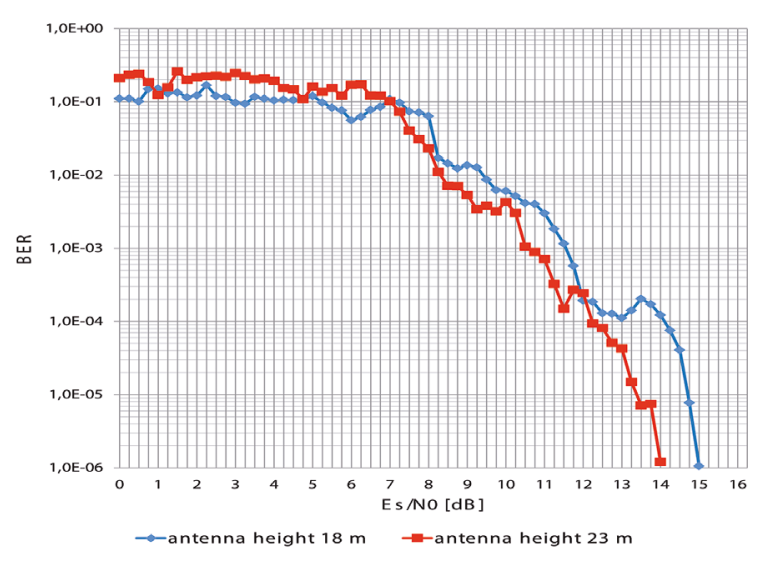

Fig. 22. BER vs. Es $/ N_{0}$ for MCS-5 and bandwidth $100 \mathrm{kHz}$ 
One of the main tasks assigned to the National Institute of Telecommunications in the EfficienSea 2.0 project was participation in technical standardization of the VDES system (mainly through cooperation with IALA). All the results presented in the paper were obtained as part of this cooperation, therefore it can be emphasized that they substantially influenced the present form of the VDES standard.

The results presented in the article clearly show that the VDE-TER ensures a large coding gain, which significantly improves the quality of data transmission and increases the bit rate. The VDE-TER provides the useful data rates of up to $200 \mathrm{~kb} / \mathrm{s}$, which is an important improvement, compared to the currently existing systems (e.g. AIS).

It should be noted that, during the measurements, a zerobit error rate for each MCS and bandwidth considered was achieved.

In the case of LOS (line-of-sight) scenarios, i.e. before crossing the radio horizon, the measured useful ranges were generally consistent with the theoretical results obtained using the ITU-R P.1546-5 propagation model. Small discrepancies between the theoretical and measured data in this case could be due to:

- man-made noise (ITU-R P.372-8) [13],

- interference with the legacy systems onboard the ships.

In the LOS scenarios, the range of the system is mainly limited by the receiver's sensitivity and the AWGN noise. After crossing the horizon line, the NLOS (non-line-of-sight) becomes the main mechanism and in this case, the observed differences between the theoretical and measured data were much more substantial than for the LOS.

In the NLOS case, the range is mainly limited by the fading (it is not AWGN any more). This is the major reason of significant discrepancies observed between the theoretical and actual ranges. Even though the propagation model ITU-R P.1546-5 takes into account the increased attenuation due to crossing the horizon line, it does not include inevitable signal fadings that occur in the NLOS environment.

It should also be observed that in the NLOS case, the MCS -5 cannot be utilized any more, and the MCS- 1 remains the only option.

Generally, the obtained results fall into „the area” between the theoretical results for the AWGN channel and the Rayleigh channel.

At this point, it should also be recalled, that VDES - in its final version - should also include a satellite segment [17]. At this point however, the issue of frequency allocations for that purpose is a source of controversy in many countries. Thus, any decisions in that respect have been postponed until the World Radiocommunication Conference WRC-19 which will take place in autumn 2019 in Sharm El Sheikh, Egypt.

\section{REFERENCES}

1. Bronk K., Mazurowski M., Rutkowski D., Wereszko B.: Simulation Investigations of the Physical Layer for a VDES System's Terrestrial Component (in Polish). Przegląd
Telekomunikacyjny i Wiadomości Telekomunikacyjne, No. 6/2016, pp. 467-470.

2. Busi R.: High Altitude VHF and UHF Broadcasting Stations. Technical Monograph, p. 3108-1967, 1967.

3. Chen Q., Song Y., Hu F., Cai C.: A Novel Method for Adjusting Digital Predistortion with Modulation Error Ratio in OFDM System. 9th International Congress on Image and Signal Processing, BioMedical Engineering and Informatics (CISPBMEI), 2016.

4. EfficienSea 2 project official website: $h t t p: / / w w w . e f f i c i e n s e a 2$. org/.

5. ETSI Technical Report ETR 290: Digital Video Broadcasting (DVB); Measurement guidelines for DVB systems, 1997.

6. General Lighthouse Authorities, Report No: RPT-09-JSa-14: VDES Channel Sounding Campaign, Safar Jan, 2015.

7. Gong M., Chen F., Yu H., Lu Z., and Hu L.: Normalized adaptive channel equalizer based on minimal symbol-errorrate, IEEE Transactions on Communications, vol. 61, no. 4, pp. 1374-1383, 2013.

8. IALA Guideline G1139: The Technical Specification of VDES, Edition 1.0, December 2017.

9. IMO, Report MSC 85/26/Add.1 Annex 20: Strategy for the Development and Implementation of enavigation, 2008.

10. ITU-R Recommendation M.1371-5: Technical characteristics for an automatic identification system using time division multiple access in the VHF maritime mobile frequency band, 2014.

11. ITU-R Recommendation ITU-R P.1546-5: Method for pointto-area predictions for terrestrial services in the frequency range $30 \mathrm{MHz}$ to $3000 \mathrm{MHz}$. Radiowave propagation, 2013.

12. ITU-R Recommendation M.2092-0:Technical characteristics for a VHF data exchange system in the VHF maritime mobile band, 2015.

13. ITU-R Recommendation P.372-8: Radio noise. Radiowave propagation, 2003.

14. Mark J. W., Zhuang W.: Wireless Communications and Networking. Pearson, 2003.

15. Molisch A. F.: Wireless Communications, Wiley, 2005

16. Proakis J.: Digital Communications, McGraw Hill, 2000

17. Raulefs R., Stenbock T. A., Dammann A., Plass S.: Physical Layer Design Towards VHF Data Exchange (VDE) 
Link, German Aerospace Center (DLR), Institute of Communications and Navigation.

18. Robertson P.: Optimal Frame Synchronization for Continuous and Packet Data Transmission. Dissertation, 1995.

19. Seybold J.S.: Introduction to RF Propagation, Wiley, 2005

20. Woodard J. P., Hanzo L.: Comparative Study of Turbo Decoding Techniques: An Overview, IEEE Transactions on Vehicular Technology, vol. 49, no. 6, November 2000.

\section{CONTACT WITH THE AUTHORS}

Krzysztof Bronk

e-mail:K.Bronk@itl.waw.pl

National Institute of Telecommunications Wireless Systems and Networks Department Jaskowa Dolina 15 80-252 Gdansk

Poland 